\title{
MINIMUM UNCERTAINTY STATES USING $n$-DEPENDENT ANNIHILATION OPERATOR
}

\author{
N. UlLah
}

Tata Institute of Fundamental Research, Colaba, Bombay 400 005, India

(Received September 5, 1994; revised version December 7, 1994)

We develop a formalism to obtain minimum uncertainty states using $n$-dependent annihilation operator. The formalism is applied to a three-dimensional harmonic oscillator and also to the one containing centrifugal barrier term. Our formalism shows that in both the cases the minimum uncertainty state can be written down as a product of the ground state and a simple function.

PACS numbers: 03.65.Ca

\section{Introduction}

In the last ten years there has been tremendous interest in the study of quantum mechanical states which give minimum value to the uncertainty product [1]. Several methods [2] have been proposed to obtain such states. The simplest being the annihilation operator method, in which one first constructs the step-down operator which gives the state $|n-1\rangle$ operating on the state $|n\rangle$ of the system. The minimum uncertainty state is then obtained by solving the eigenstate of the annihilation operator. In several problems of physical interest it happens that the annihilation operator depends on $n$. The purpose of the present work is to develop a formalism to construct minimum uncertainty states using such annihilation operators. We shall use the three-dimensional harmonic oscillator to bring out the essential features of the present formalism, which is given in the next section. Concluding remarks are presented in Sec. 4.

\section{Formulation}

We would first like to describe briefly the one-dimensional harmonic oscillator whose eigenvalue equation is given by

$$
\left(-\frac{1}{2} \frac{\mathrm{d}^{2}}{\mathrm{~d} x^{2}}+\frac{1}{2} x^{2}\right) \phi_{n}=\left(n+\frac{1}{2}\right) \phi_{n},
$$


where we have used the units $\hbar=m=k=1$ and the eigenfunctions $\phi_{n}$ are given by

$$
\phi_{n}=\pi^{-\frac{1}{4}}\left(2^{n} n !\right)^{-\frac{1}{2}} H_{n}(x) \exp \left(-\frac{1}{2} x^{2}\right),
$$

$H_{n}(x)$ being Hermite polynomials [3].

The step-down operator is given by

$$
\frac{1}{\sqrt{2}}(x+\mathrm{i} p) \phi_{n}=\sqrt{n} \phi_{n-1} \text {, }
$$

where $p=-\mathrm{i} \frac{\mathrm{d}}{\mathrm{d} x}$.

The minimum uncertainty state $\chi(x)$ is the solution of the equation

$$
\frac{1}{\sqrt{2}}(x+\mathrm{i} p) \chi=\lambda \chi,
$$

where $\lambda$ in general is taken to be complex. In writing the solution of the eigenvalue equation of the annihilation operator, one imposes the condition that the state $\chi$ must contain ground state. This condition will become important when we consider the three-dimensional case later.

The solution of Eq. (4) is straightforward, it is given by

$$
\chi(x)=A \exp \left(-\frac{1}{2} x^{2}+\sqrt{2} \lambda x\right),
$$

where $A$ is the normalization constant.

We shall now consider the three-dimensional oscillator, where the annihilation operator becomes $n$-dependent. We shall be concerned with the $s$-wave radial equation which is given by

$$
\left[-\frac{1}{2} \frac{\mathrm{d}^{2}}{\mathrm{~d} r^{2}}-\frac{1}{r} \frac{\mathrm{d}}{\mathrm{d} r}+\frac{1}{2} r^{2}\right] R_{n}(r)=\left(2 n-\frac{1}{2}\right) R_{n}(r),
$$

where the radial eigenfunctions $R_{n}(r)$ are given by

$$
R_{n}(r)=(-1)^{n-1} \pi^{-\frac{1}{4}} 2^{-n+1}[(2 n-1) !]^{-\frac{1}{2}} \frac{1}{r} H_{2 n-1}(r) \exp \left(-\frac{1}{2} r^{2}\right),
$$

and $n=1,2, \ldots$ It is easy to show that the step-down operator is now given by

$$
\left[r \frac{\mathrm{d}}{\mathrm{d} r}+r^{2}-2(n-1)\right] R_{n}=-\sqrt{2(2 n-1)(n-1)} R_{n-1},
$$

and is $n$-dependent.

The main point of the present formulation is that the $n$-dependence of the step-down operator can be written in terms of differential operator, which in the present case is given by expression (6). We can therefore write the following equation using (6), (8) to determine the minimum uncertainty state $\chi$,

$$
\left[\frac{1}{2} \frac{\mathrm{d}^{2}}{\mathrm{~d} r^{2}}+\left(r+\frac{1}{r}\right) \frac{\mathrm{d}}{\mathrm{d} r}+\frac{1}{2} r^{2}+\frac{3}{2}\right] \chi(r)=\lambda \chi(r) .
$$

In order to solve this equation, we first write

$$
\chi(r)=\frac{1}{r} \psi(r)
$$


which gives

$$
\left[\frac{1}{2} \frac{\mathrm{d}^{2}}{\mathrm{~d} r^{2}}+r \frac{\mathrm{d}}{\mathrm{d} r}+\frac{1}{2} r^{2}+\frac{1}{2}\right] \psi=\lambda \psi
$$

This can be re-written as

$$
\left(\frac{\partial}{\partial r}+r\right)^{2} \psi(r)=2 \lambda \psi(r)
$$

In order to solve Eq. (12) we first solve the following equation:

$$
\left(\frac{\partial}{\partial r}+r\right) \phi(r)=w \phi(r)
$$

which can be easily solved to give

$$
\phi(r)=A \exp \left(-\frac{1}{2} r^{2}+w r\right)
$$

where $A$ is a constant.

Applying the operator $\left(\frac{\partial}{\partial r}+r\right)$ on Eq. (13) and comparing the resulting equation with (12), we find

$$
w^{2}=2 \lambda \text {. }
$$

Remembering that the solution to (12) must contain the ground state $R_{n=1}$, we finally get

$$
\psi(r)=A[\exp (\sqrt{2 \lambda} r)-\exp (-\sqrt{2 \lambda} r)] \exp \left(-\frac{1}{2} r^{2}\right)
$$

Thus the minimum uncertainty state $\chi(r)$ for the three-dimensional harmonic oscillator is given by

$$
\chi(r)=\frac{A[\exp (\sqrt{2 \lambda} r)-\exp (-\sqrt{2 \lambda} r)]}{r} \exp \left(-\frac{1}{2} r^{2}\right) .
$$

To calculate the value of the uncertainty product, one could now calculate the expectation values of the operator $p_{\mathrm{r}}$ corresponding to radial momentum and also of the radial coordinate $r$ using the wave function $\chi$ given by (17). Writing $q=\sqrt{2 \lambda}$, one finds, e.g., for $r$ :

$$
\begin{aligned}
& \langle\chi|r| \chi\rangle=\left[\exp q_{\mathrm{r}}^{2}-\exp \left(-q_{\mathrm{i}}^{2}\right)\right]^{-1}\left[\left(q_{\mathrm{r}} \operatorname{er} f q_{\mathrm{r}}\right) \exp \left(q_{\mathrm{r}}^{2}\right)\right. \\
& \left.\quad+\mathrm{i} q_{\mathrm{i}} \operatorname{er} f\left(-\mathrm{i} q_{\mathrm{i}}\right) \exp \left(-q_{\mathrm{i}}^{2}\right)\right]
\end{aligned}
$$

and similar expressions for $\left\langle r^{2}\right\rangle,\left\langle p_{\mathrm{r}}\right\rangle$ and $\left\langle p_{\mathrm{r}}^{2}\right\rangle$, where $q=q_{\mathrm{r}}+\mathrm{i} q_{\mathrm{i}}$ and \langle\rangle denotes the expectation value and er $f$ denotes the error function.

From these expressions we find that if $|q| \rightarrow \infty$, the product $\left[\left\langle r^{2}\right\rangle-\langle r\rangle^{2}\right]$ $\times\left[\left\langle p_{\mathrm{r}}^{2}\right\rangle-\left\langle p_{\mathrm{r}}\right\rangle^{2}\right] \rightarrow \frac{1}{4}$, the minimum value. 


\section{Harmonic oscillator with centrifugal barrier}

As a further application of the formulation described in Sec. 2, we apply it to the Hamiltonian $H$ having an oscillator potential and a centrifugal barrier term. It is given by

$$
H=-\frac{1}{2} \frac{\mathrm{d}^{2}}{\mathrm{~d} r^{2}}-\frac{1}{r} \frac{\mathrm{d}}{\mathrm{d} r}+\frac{1}{2} r^{2}+\frac{\lambda}{r^{2}},
$$

where $\lambda$ is a positive constant.

The exact eigenfunctions of the Hamiltonian can be written down in terms of confluent hypergeometric functions. Using the steps given in Sec. 2, we find that the minimum uncertainty state $\chi$ for this system is given by

$$
\chi=(\text { const. }) r^{-\frac{1}{2}}\left[\exp \left(-\frac{1}{2} r^{2}\right)\right] I_{\sqrt{\frac{1}{4}+2 \lambda}}(\sqrt{2 \alpha r}),
$$

where $\alpha$ is a complex parameter and $I_{\mu}$ is the modified Bessel function.

We note from expressions (17) and (20) that in both the cases the minimum uncertainty state is a product of the ground state wave function and another function like Sinh or modified Bessel function. This can be very useful when one wants to find minimum uncertainty states for the potentials for which exact analytic eigenfunctions cannot be written down.

\section{Concluding remarks}

We have shown how to construct the minimum uncertainty states using $n$-dependent annihilation operator. An explicit expression for the minimum uncertainty state for the three-dimensional oscillator and one having centrifugal barrier is given.

We remark here that Nieto and Simmons, Jr., had also discussed [2] the $n$-dependence of the raising and lowering operators. In their work they had used the recursion relation to arrive at the minimum uncertainty state using harmonic oscillator with barrier as an example. Their final solution was obtained by comparing the expansion coefficients with the already derived state for the barrier using quantum equation of motion, while here we have directly obtained the differential equation and solved it with the condition that ground state of the three-dimensional oscillator be obtained as a special case of the solution of the differential equation.

As remarked in Sec. 3, our formulation can also be used to obtain approximate minimum uncertainty states for potentials for which exact analytic eigenfunctions cannot be found.

\section{References}

[1] J.R. Klauder, B.-S. Skagerstam, in: Coherent States - Applications in Physics and Mathematical Physics, World Scientific, Singapore 1985.

[2] M.M. Nieto, L.M. Simmons, Jr., Phys. Rev. D 20, 1332, 1342 (1979); M.M. Nieto, D.R. Truax, Phys. Rev. Lett. 71, 2843 (1993) (and other references cited in this paper).

[3] L.I. Schiff, in: Quantum Meçhanics, McGraw-Hill, New York 1955. 